\title{
Tecno-patrones: Pieles, contexto y fabricación
}

\section{Carmelo Zappulla}

DOl: 10.5821/palimpsesto.11.3700

\section{Introducción}

La concepción del proyecto no se puede separar de su materialización: a menudo es la técnica la que ayuda al desarrollo formal del proyecto y constituye su misma esencia. Por lo tanto, pone sus raíces en la etimología y en el rol que ha asumido en la filosofía.

Desde la época de Platón, según Heidegger, había una fuerte relación entre epistêmê y technê, donde ambas palabras se en el sentido más amplio. La techne nocimiento teórico. Es una teoría que se convierte en práctica, es concreta y depende del contexto'.

En 'The Question Concerning Technology', Heidegger propone que la technê no reside principalmente en la fabricación o en que la technê no reside principalmente en la fabricación o en el uso de herramientas, sino en la revelación. La tecnología en el proceso de creación, que transforma la materia y genera interconexiones. Así que la tecnología no es sólo una herramienta, sino una forma de revelar y de saber ${ }^{2}$.

En este artículo hablaremos de tecnología en términos de revelación de la materialidad, de cómo es parte del proceso creativo de diseño

Con 'revelación de la materialidad' entendemos, por tanto, no sólo la expresión de una arquitectura honesta que expresa claramente la forma en la cual se construye, sino de un proceso de interacción entre diferentes niveles: actividad humana, herramientas, materia, forma, estructura, medio ambiente y comunicación Asl, parece razonable poner la «revelacion" dentro de un marco sistémico que nos ayude a percibirla como consecuencia de un cionar y orientarla a la generación de una estructura material.

Pero entonces, ¿cómo interactúan los diferentes niveles que subyacen en la materialidad del proyecto?

subyacen en la materialidad del proyecto?
Éstos interactúan a través del patrón, aquella 'configuración Estos interactúan a través del patrón, aquella 'configuración abstracción con la manifestación matérica. El patrón es aquel dispositivo visualmente gráfico y al mismo tiempo abstracto que se centra en el aspecto cualitativo de la materialidad y de su organización

Volver a la etimología de los términos abre interesantes relaciones entre el concepto de Patrón y el de Materia. La etimología de la palabra patrón se debe probablemente al griego, Pater, padre, entendido como aquel que engendra y da estructura a la familia. El patrón es entonces lo que conecta, lo que relaciona las diferentes partes de una estructura compuesta. Es curioso, en cambio, cómo la etimología de la palabra Materia derive del latín Mater, madre, primera sustancia de la que están formados los demás, todo lo que tiene forma, cuerpo y ocupa el espacio. De la relación entre Mater y Pater, o entre Patrón y Materia, podemos explicar la generación de la arquitectura o, como en este caso en concreto, la relación entre la tecnología y la materialidad.

Está claro que, inevitablemente, el trabajo sobre la materia tiene una implicación directa en el desarrollo de patrones; un orden, que pertenece al construir, emerge en el proceso de construcción o se fortalece conscientemente en la fase de diseño. Así es como los avances en la tecnología afectan a la forma, a los materiales en la forma de ensamblarlos, y la construcción de la propia arquitectura.

El patrón, sin embargo, no se impone como un orden ajeno sobre la materia, sino que debe interpretar sus características morfológicas, las fuerzas que entran en juego, la capacidad performativa, etc.

Cada sistema material tiene una cierta "vocación formal:" es esta vocación de la que emergen los "tecno-pattern", es decir, los patrones propios de la materialidad, de la organización física destinada a la eficiencia técnica.

El mismo Focillon reivindica la relación fundamental entre técnica, herramientas, materiales y la creación formal: la forma sugerida por el material y por la técnica que se le impone. La materia, en este sentido, tiene el potencial de crear una correspondencia única con la obra, que se manifiesta a través de patrones de colores, texturas y geometrías: es materia que, en el acto constructivo, se encuentra profundamente transformada, estableciendo un nuevo orden distinguible de la materia prima "La madera de la estatua no es la madera del árbol"”, es un material que lleva las marcas del proceso, de las herramientas que le han dado forma, dejando la impronta de un nuevo orden: "La vida de la materia se ha transformado"5.

Volviendo más específicamente a la arquitectura y al proceso de diseño, se puede afirmar que existe un diálogo entre las fases de generación espacial y de definición material. Esta última influencia a la primera y viceversa, en un circuito de retroalimentación en el que la materialización modifica el espacio y el espacio la materia.

En la práctica arquitectónica es posible reconocer dos actitudes: en la primera, la experimentación formal anticipa una racionalización constructiva posterior; en la segunda, por el contrario, la experimentación formal es consecuencia o es intrínseca a la misma experimentación material.

En ambos casos, el espacio siempre será sometido a una sistematización constructiva, orientada a la identificación de sus elementos técnicos y materiales. Detrás de la arquitectura existe
siempre un tecno-patrón que se genera según principios estéti-

\section{Envolvente, de la indiferenciación a la adaptabilidad}

Llegados a este punto, nos enfrentamos al tema de la piel de la arquitectura, del límite que separa el interior del exterior, de aquel complejo confín que distingue y vincula la vida del hombre y su entorno.

El revestimiento de los edificios es el elemento arquitectónico más primitivo, que al mismo tiempo protege la propiedad privada y está expuesto al ambiente externo: la piel dialoga entonces con los elementos atmosféricos, defendiéndose de la luvia, del calor y del frío, controlando el paso de la luz, del aire y orientando las visuales hacia el exterior. Esta piel encierra el espacio interior en las tres dimensiones y posee una importancia no sólo tecnológica, sino también ecológica, política y de representatividad.

La conciencia sobre las cuestiones medioambientales ha despertado el interés sobre el consumo energético de los edificios. La piel exterior, por ejemplo, ya no puede ser una superficie de vidrio indiferenciada que crea un efecto invernadero luego contrarrestado por aire acondicionado: es necesario estudiar una pie que responda a la orientación del sol, que proteja en verano y deje pasar la luz en invierno. En los seres humanos, la piel regula naturalmente la temperatura en nuestro cuerpo, lo que representa una defensa con respecto a los cambios meteorológicos, y funciona a través de una serie de dispositivos que reaccionan moamicamente a las condiciones climáticas externas. Del mismo modo, en el ámbito arquitectónico "la piel es una membrana homeostática que regula los flujos de energía entre el exterior y el cada vez más en el estudio de las superficies externas, que deben cumplir con parámetros ambientales específicos.

Volviendo más específicamente a la arquitectura y al proceso de diseño, se puede afirmar que existe un diálogo entre las fases de generación espacial y de definición material. Esta última influencia a la primera y viceversa, en un circuito de retroalimentación en el que la materialización modifica el espacio y el espacio la materia.

\subsection{Inmaterialidad indiferenciada}

Previo a la difusión del aire acondicionado, los muros de los edificios eran gruesos y las aberturas tenían una dimensión reducida, lo que permitía aislar térmicamente los edificios.

En la primera mitad de 1900, los efectos de la Revolución Industrial se difundieron ampliamente y la transparencia se convirtió en una cualidad inherente a la arquitectura.

El Crystal Palace de la Exposición Universal de Londres 1851 es el arquetipo de los modernos avances tecnológicos aplicados a la fabricación de vidrio y acero fundido, avances que se tradujeron en condiciones imprescindibles para la arquitectura moderna y contemporánea: grandes visuales, luz, aire e inmaterialidad.

De hecho, estas tecnologías, que en un principio pertenecían sólo a los ingenieros, se van extendiendo entre los arquitectos. Mies Van de Rohe sublimó la misma idea de transparencia, logrando en la Farnsworth House (Plano, 1950), una arquitectura casi inmaterial.

Una nueva interpretación de la transparencia fue experimentada por Wright en la Torre Johnson Wax Laboratory (Racine, 1950). A través de un proceso de redundancia material, se produce una seriación lineal de tubos de vidrio que sustituye las ventanas tradicionales. La transparencia total se convierte en un efecto borroso y difuso del entorno.

En los últimos años, la técnica ha permitido superar la distinción entre paredes, techos y ventanas: actualmente es posible utilizar el mismo tratamiento para todas las superficies exteriores de edificio, destacando así su carácter objetual.

Un ejemplo es el diamante Prada (Tokio, 2003), de Herzog \& De Meuron. Este cristal icónico tiene una fachada de vidrio, constituida por un patrón romboidal de paneles planos, cóncavos y convexos. El patrón romboidal incorpora no sólo los paneles de vidrio, sino también la estructura y unas áreas funcionales visibles desde el exterior. Así es como este patrón integra diferentes niveles: estructura, fachada, estética, representatividad y distribución funcional.

Otra arquitectura de fuerte carácter objetual en Tokio es la sede de Dior de Sanaa. El edificio, que desde la distancia parece un prisma transparente, de cerca tiene una densidad más compleja, producto de la superposición de dos superficies, el vidrio exterior y

\subsection{Patrón responsivo estático}

El himno a la transparencia ha conferido, por un lado, calidad estética e higiene; por otro ha provocado la introducción masiva de sistemas de acondicionamiento térmico, contribuyendo al calentamiento global del planeta.

La objetualidad que caracteriza los casos anteriores, si por un lado afirma una imagen escultórica, por el otro lleva a destacar una cierta indiferenciación responsable de una relación controvertida entre la arquitectura y el contexto. El uso indiferenciado de un material o revestimiento entra muchas veces en contradicción con las características no homogéneas del contexto

que requieren un

El patrón, por lo tanto, representa un posibilidad por parte de la piel arquitectónica de mantener el carácter objetual y al mismo tiempo interactuar con el contexto circundante, tamizando la luz, la irradiación solar, las visuales.

Recientemente, en un contexto en el cual el debate sobre el clima se vuelve imperativo, la arquitectura ha respondido tratando de aumentar la eficiencia energética de los edificios. Esto representa para los arquitectos, y en general para el mundo de la construccion, una oportunidad para generar pieles multicapa, más o menos permeables, con soluciones tecnológicas diferenciadas, y ha dado lugar a un campo de aplicacion vastisimo que per-
mite desarrollar una amplia variedad de sistemas de protección de fachada.

Se abandona la diferenciación de los materiales en relación con las funciones propias del movimiento moderno, y se conciben pieles cada vez más inteligentes que combinan varios aspectos del proyecto en lugar de separarlos: ecológicos, comunicativos y representativos.

Por ejemplo, Herzog \& De Meuron están entre los arquitectos más prolíicos en la concepción de patrones que refuerzan la objetualidad de la arquitectura y al mismo tiempo incluyen más componentes del proyecto. Muy probablemente les pertenece el primer caso de piel resultado de un patrón reactivo. Se remonta a 1994, en el Signal Box Aufdem Wolf, Basilea. El edificio de hormigón está revestido de bandas horizontales de cobre que, a través de una torsión gradual, permiten el paso desde la opacidad hacia la transparencia. El revestimiento funciona como una jaula de Faraday: es capaz de aislar el interior de cualquier campo electrostático externo. Así, dentro del mismo sistema, es posible integrar con un simple patrón de aislamiento electrostático la opacidad y la transparencia.

Después de un proceso de diseño que se prolongó durante casi veinte años, el enfoque de los arquitectos suizos se mantiene inalterado en el reciente Messe Basel- New Hall (2013). Igualmente aquí la envolvente está constituida por dos capas. La exterior es una superficie de aluminio, que incorpora el patrón de las mallas déployée: la malla se escala gradualmente y genera un gradiente que va desde lo opaco hasta lo permeable. Se producen efectos esfumados que controlan la penetración de la luz, sin interrupciones bruscas o cambios de materiales. No se contempla superponer diferentes órdenes, y el sistema constructivo.

Hemos visto en estos y otros casos de Herzog y De Meuron un trabajo desarrollado en dos estratos, una piel interna convencional y una externa que le confiere la imagen final del edificio y controla de manera difusa la luz. De una manera sustancialmente diferente, UNStudio y Zaha Hadid profundizan en la integración de la transparencia en la teselación de la piel. Recordemos, de hecho, que algunos de sus proyectos, de manera similar, generan un patrón que se compone de módulos que, cambiando el tamaño y la profundidad, permiten a través de una transformación progresiva el paso de la luz.

Recordemos en primer lugar el proyecto de Z. Hadid, los Juz-

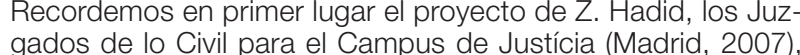
gados de lo Civil para el Campus de Justícia (Madrid, 2007), cual se insertan elementos abiertos, parcialmente abiertos, pequeños aleros a sur, este y oeste. Por otra parte, dentro de mismo sistema, estos elementos cambian sus características mismo sistema, estos elementos cambian sus caracteristicas progresivamente, a fin de pasar de un elemento totalmente abierto a ur

UNStudio trabajó en la misma dirección en el proyecto para el concurso del centro comercial Omotesando (Tokio, 2008). En este caso, la variación de la permeabilidad se confía a una teselación hexagonal que manifiesta un tejido entrelazado. La reducción de las aberturas es, en este caso también, conde su perímetro.

\subsubsection{Patrón responsivo cinemático: mecánico Vs Material}

La capacidad responsiva que hemos estudiado en los ejemplos anteriores se basa en la variación de estructuras geométricas estáticas, fundada en la transición progresiva de la permeabilidad de la piel en función de la orientación del sol y de las visuales. Ahora consideraremos la permeabilidad que involucra sistemas móviles que reaccionan a los estímulos externos con el movi-miento. Un sistema de sombreado de las fachadas se basa en el uso de elementos móviles: membranas articuladas, controladas mediante software, regulan el paso de la luz en función de la exposición solar.

La primera operación de este tipo fue estudiada y aplicada por Jean Nouvel en el Institut du Monde Arabe (1981-1987). La fachada 'árabe' se compone de 30.000 diafragmas sensibles a la luz, con el fin de regular la insolación a través de un sistema mecanizado, controlado por sensores, que filtra los rayos solares. Teniendo en cuenta que el proyecto se remonta a 1981 , la solución es extraordinariamente innovadora en relación tanto a la tecnología como al significado cultural que establece con su entorno.

Hubo otros intentos de este tipo, como el sistema neumático desarrollado por Foiltec en el proyecto del Atelier Brückner para la Expo 2000 de Hannover ${ }^{7}$ o las residencias sociales de E. François Louviers en Francia (2006). 
La idea de crear fachadas dinámicas capaces de regular la insolación del edificio ha sido incluso patentada por Tessellate ${ }^{\mathrm{TM}}$. lación del edificio ha sido incluso patentada por Tesselate paneles perforados, en el que el deslizamiento de uno respecto paneles perforados, en el que el deslizamiento

La introducción de un sistema mecánico a menudo implica costosos problemas de mantenimiento que, como ha ocurrido en el Institut du Monde Arabe, pueden terminar renunciando a la interactividad de la fachada.

Nuevos estudios sobre materiales permiten la sustitución de complejos dispositivos mecánicos por sistemas materiales que responden automáticamente a los estímulos externos, sin la necesidad de electricidad, sensores $u$ otros dispositivos. En particular, D.K. Sung, siguiendo el paralelismo entre la piel humana y la de los edificios, está examinando la posibilidad del bimetal térmico, un material laminado que se dobla cuando se calienta. Su investigación se basa en el desarrollo de superficies que permiten controlar la entrada de luz a través de la deformación de
sus elementos constitutivos. Esto sucede sin el uso de energía externa, sólo gracias a la propia naturaleza del material.

Similar es la investigación llevada a cabo por Achim Menges y Steffen Reichert, que estudia la capacidad responsiva a la humedad de sistemas materiales constituidos por láminas de madera.

Se abandona la diferenciación de los materiales en relación con las funciones propias del movimiento moderno, y se conciben pieles cada vez más inteligentes que combinan varios aspectos del proyecto en qugar de separarlos: ecológicos, comunicativos y representativos.

\section{El patrón de la aproximación geométrica. Superficies} complejas y fabricación digital

Permaneciendo en el ámbito de las pieles arquitectónicas, paralelamente al desarrollo de software capaces de modelar y gestionar superficies complejas, se han desarrollado sistemas de producción de control numérico. En este proceso se instauran métodos de aproximación de "superficies matemáticas" que, en la fase de producción, se transforman, a través del ensamblaje la fase de produccion, se transforman, a traves del ensa serie de paneles, en superficies discretizadas.

de una serie de paneles, en superficies discretizadas. La triangulación es uno de los métodos más estudiados, ya que ficie compleja en un conjunto de triángulos planos. La aproximación en este caso es muy baja, a menos que se utilicen panemación en este caso es muy baja, a menos que se utilicen paneles muy pequeño

de elementos.
El teatro de los títeres de M. Meredith para el Carpenter Center en Cambridge es un ejemplo de discretización de una superficie compleja en una serie de paneles triangulares/ romboidales. La fabricación es digital, el diseñador prepara los archivos que la máquina corta directamente. Dado que el proceso de construcción se acorta hay menos intermediarios, menos posibilidades de re-interpretar el proyecto y multiplicar los errores.

Hay que señalar que la triangulación ha generado su propia estética absolutamente independiente, que prescinde de la misma aproximación de superficies complejas. Muchos proyectos están, de hecho, diseñados por triangulación desde los primeros bocetos, principalmente por razones proyectuales y no sólo económicas.

Sin embargo, la investigación sobre aproximaciones geométricas no termina con la triangulación y la difusión de las superficies free-form, sino que ha impulsado a varios investigadores a desarrollar sistemas de racionalización más sofisticados que descartan a priori la triangulación, optando por paneles cuadriláteros. Por ejemplo, H. Pottmann ${ }^{8}$ demuestra cómo una superficie compleja puede ser construida en función de sus "necesidades geométricas", adoptándose simultáneamente paneles planos, a simple o doble curvatura, optimizando significativamente los costes de producción. Algunas superficies, como las regladas, pueden ser construidas a través de moldes económicos de poliestireno, como en el Centro de Arte Contemporáneo de Z. Hadid (Cagliari, 2007) ${ }^{9}$

Una manera sorprendente de aproximación ha sido concebida por M. Fornes: algunos de sus proyectos como el non Lin/LinPavilion revelan una ingeniosa estrategia constructiva basada en la división en tiras desplegables de superficies altamente complejas. Por lo tanto, las tiras con las cuales el modelo virtual ha sido aproximado, pueden ser desplegadas virtualmente y sucesivamente cortadas a control numérico y dobladas durante el montaje.

\subsection{Fabricación digital}

Como hemos visto, el uso de máquinas a control numérico (CNC) y de otros sistemas de producción digital ha jugado un papel fundamental tanto en la manipulación algorítmica del proyecto como en la aplicación y fabricación de geometrías complejas. En particular, la introducción de máquinas que fabrican directamente parte del proyecto implica varios aspectos a considera

- Pensar el proyecto en función de su posible racionalización y construcción digital. Esta fase implica a menudo replantear y
redefinir, por ejemplo, la configuración y la aproximación de una redefinir, por

- La preparación de un archivo especial de construcción, un proceso de descomposición del proyecto orientado a la producción directa de sus partes.

- Se acorta la distancia entre arquitecto y constructor. El archivo se prepara a escala real y está pensado para ser directamente fabricado.

Resulta imprescindible dedicar unas palabras a la descripción de las técnicas de fabricación digital utilizadas en arquitectura debido a que, como hemos afirmado al principio del capítulo, cada una de las siguientes tipologías está estrechamente relacionada con el proceso físico del proyecto.

Como sabemos, existen dos procesos de fabricación digital que se diferencian por la manera en que se manipula la materia constructiva:

1. El proceso sustractivo en el cual se fresa, corta o graba un material. Tal proceso tiene que ver principalmente con máquinas de corte: al agua y láser, o fresadoras clasificadas según el número de ejes que poseen

2. El proceso aditivo consiste, al contrario, en construir acumulando material. Aunque, por lo general, se refiere a la impresión 3D de objetos de pequeña escala como la FDM (fused deposition modeling) o la las SLS (selective laser sintering), E. Dini ha adaptado esta tecnología a la gran escala.

Transversalmente a estos sistemas de fabricación, también los robots industriales han recibido un gran interés en el campo arquitectónico. Estos dispositivos, a diferencia de los anteriores, no están especializados en una sola tarea, sino que se pueden programar y personalizar para desarrollar los sistemas materiales mas inesperados. Además, por su propia naturaleza de brazo mecánico, son capaces de moverse y llegar a cualquier punto dentro de un determinado dominio espacial. Se prestan, por lo tanto, no sólo a desarrollar todas las tareas ya realizadas por las máquinas de corte CNC, sino también a seguir de manera extraordinaria procesos particulares de montaje.

En la Universidad de Stuttgart, el Institute for Computational Design fundado por Achim Menges, cuenta con un laboratorio de fabricación robótica y desde el 2010 trabaja en la investigación computacional orientada a la construcción de pabellones que involucran la programación de brazos robóticos. Esto condujo a la definición de nuevos sistemas materiales como en el caso de Winding T, 2013-14, un pabellón donde los robots tejen módulos de fibra de carbono reforzada sin el uso de moldes. Asimismo, el laboratorio de investigación Dfab, dirigido y fundado por Gramazio y Kohler en la ETH (Zurich), ha dado sus frutos en numerosos experimentos que han utilizado robots industriales en la construcción de prototipos utilizando la manipulación de ladrillos.

La Programmed Wall (ETH Zurich, 2006) muestra cómo se puede enseñar a la máquina a construir una superficie compleja a través del ensamblaje de ladrillos convencionales, donde los estudiantes definen la lógica constructiva con la que el material va a ser organizado ${ }^{10}$. La misma investigación ha sido aplicada a escala arquitectónica en la fachada de la bodega Gantenbein (Fläsch, 2006)

Recientemente, en la investigación del Flight Assembled Architecture (FRAC, Centre Orléans, 2011-2012), han llegado a hipotetizar sobre la construcción de un rascacielos realizado por 'cuadricópteros', capaces de colocar "bloques" en el espacio, superando por lo tanto la restricción espacial que poseen los brazos robotizados. Estos proyectos representan un reto que centra la atención en la computienen raíces antiquísimas.

La fabricación digital of rece la posibilidad de volver a experimentar aquella complejidad, sustituyendo la capacidad artesanal perdida: en otras palabras, la máquina puede reproducir dinámicas de fabricación o montaje.

La articulación de bloques como el ladrillo ha estimulado la composición de patrones extremamente interesantes desde los tiempos romanos. Por otra parte, la arquitectura arabe integra a través de la compleja disposición de ladrillos, patrones ornamentales. El uso de texturas constructivas a través del ladrillo ha tenido una importancia fundamental en la historia, determinando sistemas estructurales innovadores, pero el cambio profundo que ha vivido la arquitectura hace que, hoy en día, no haya mano de obra para lograr tal complejidad.

La fabricación digital ofrece la posibilidad de volver a experimentar aquella complejidad, sustituyendo la capacidad artesanal perdida: en otras palabras, la máquina puede reproducir dinámicas de fabricación o montaje. Como en los ejemplos que hemos comentado, de Gramazio y Kohler, a través de alejamiento gradual de ladrillos es posible producir gradientes que introducen gradualmente aire y luz. Así, lo que parecía un simple dispositivo técnico en realidad puede transformarse en un instrumento de proyecto capaz de 'revelar', de relacionar la un instrumento de proyecto capaz de 'revelar', de relacionar la construcción con otros niveles de proy
estructural, el contextual y el estético.

Desde el momento en que debemos enseñar al Robot el procedimiento de construcción, estamos obligados a descifrar todas las fases de ensamblaje y traducirlas a la máquina, manteniendo un control absoluto sobre la construcción. La ventaja es la de poder explicitar el método constructivo mismo y al mismo tiempo poder entar el metodo constructivo mismo y al mismo tiempo poder entrar activa y creativamente en un proceso que, por lo general, estaba supeditado a capacidades ajenas al arquitecto. Por otro lado, sin embargo, se debe renunciar a la trabajo manual ${ }^{11}$.

\section{Conclusión}

Hemos visto cómo el estudio de la piel es una excusa para hablar acerca de la tecnología, no sólo como una herramienta para hacer, sino sobre todo para comprender, para 'revelar' creativamente. En este proceso de generación, el patrón actúa como puente entre la materia y la técnica.
El estudio de la piel es una excusa para hablar acerca de la tecnología, no sólo como una herramienta para hacer, sino sobre todo para comprender, para 'revelar' creativamente. En este proceso de generación, el patrón actúa como puente entre la materia y la técnica.

Hoy, gracias al nuevo interés hacia el medio ambiente, la importancia del revestimiento en relación con el entorno es crucial. Dentro de esta interacción, la envolvente se alimenta de otros significados, como el comunicativo, el social o el político. plejidad formal que se enfrenta a cuestiones geométricas y esplejidad formal que se enfrenta a cuestiones geométricas y estructurales particulares. La cuestión de la aproximación geométrica conduce a estudios sofisticados de la forma y la fabricación digital. Esto ha abierto nuevos caminos en la introducción de la robotica en el proceso de construcción, incluso revolucionando Tambén a pesar de que la reproducción homogénea haya imperado dua pesar de que la reproducción homogénea haya imperado
rante los doscientos años de producción industrializada.

Sólo a partir de los últimos 20 años, este sistema de producción está experimentando una importante transformación a través de la fabricación digital y la entrada del ordenador en el sistema de producción. Por un lado se reduce la distancia entre el diseñador y el objeto producido, por el otro el ordenador, entrando en el proceso de la gestión de algunos parámetros de diseño permite la generación de diversas declinaciones formales.

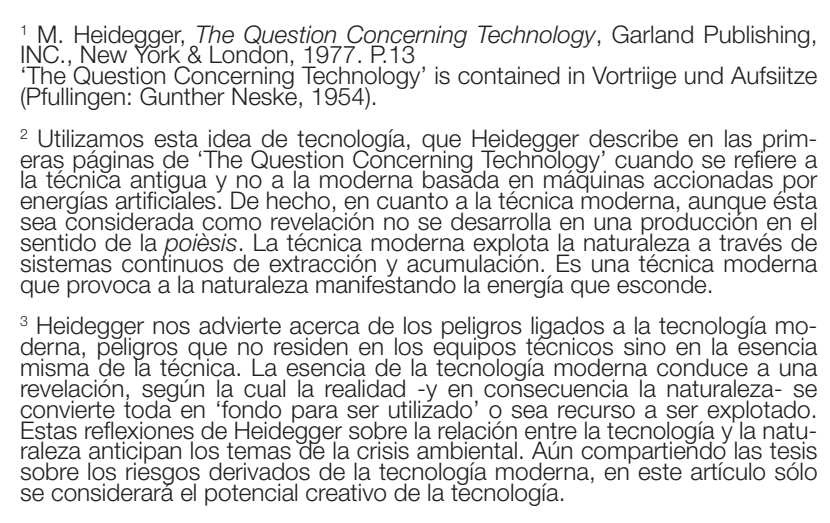
${ }^{4}$ Focillon [1990], p. 53.

${ }^{5} / \mathrm{dem}$.

6 Ver la conferencia de A. Zaera Polo "Envelopes" (19 de noviembre de 2009)
tomado de la Southern California Institute of Architecture (SCl-Arc), Los An-
geles (http://sma.sciarc.edu/video/alejandro-zaera-polo-envelopes). 7 El sistema neumático ha permitido acercar o alejar las dos membranas
de los cojines del revestimiento exterior. Una representa el patrón positivo
y lo otra el negativo, de tal manera que al acercarse entre ellos transforitan
a fachada en ${ }^{8} \mathrm{Cfr}$. Pottmann [2010], pp. 72-77.

${ }^{9}$ Cfr. Pottmann [2010], p. 74.

10 Gramazio - Kohler en: http://www.dfab.arch.ethz.ch/web/e/lehre/81
html(traduzionemia).

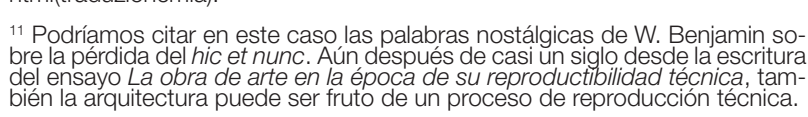

REFERENCIAS

T. Bonwetsch, "Designing robotic assemblies", en P. Terri, P. Brady, Inside
Smart Geometry. Expanding the Architectural Possibilities of Computational
Design, John Wiley \& Sons, 2013, pags. 218-231.

H. Focillon, Vita delle Forme, Piccola Biblioteca Einaudi, Torino, 1990

M. Heidegger, The Question Concerning Technology, Garland Publishing,
INC., New York \& London, 1977 . H. Pottmann, "Architectural geometry as design knowledge", The New Struc-
turalism: Design, Engineering and Architectural Technologies, 80 (4), 2010,
págs. $72-77$ A. Zaera Polo, "The politics of the envelope, a political critique of materialism",
en A. Oosterman ede.), OVlume \#17. Content Management, Stichting Archis'
Publisher, Amsterdam, 2008, págs. 76-105. C. Zappulla, Architecture as Science of Pattern? Tesis de doctorado. Barce-
lona. niversitat Politerenica de Catalunya, Departament de Projectes Arqui-
tectonics, 2014, págs. $250-287$.

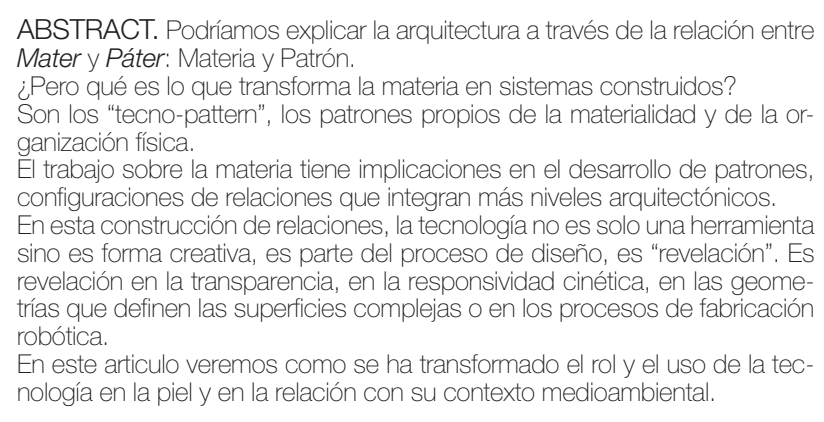

Palabras clave: materia, patrón, sistema, piel, tecnología.

Carmelo Zappulla es Doctor en arquitectura por la Universitat Politècnica de Catalunya y socio fundador de External Reference Architects. Enseña en el Instituto de Arquitectura Avanzada de Catalunya y en el Istituto Europeo di Design. 